\title{
Detection of small for gestational age in preterm prelabor rupture of membranes by Hadlock versus the Fetal Medicine Foundation growth charts
}

\author{
Jose Duncan, MD ${ }^{1,2}$, Karla Leavitt, $\mathrm{MD}^{2}$, Kristin Duncan, $\mathrm{RN}^{3}$, Gustavo Vilchez, MD \\ Department of Obstetrics and Gynecology, ${ }^{1}$ University of Tennessee Health Science Center, Memphis, TN; ${ }^{2}$ University of South Florida, Tampa, FL; \\ ${ }^{3}$ Labor and Delivery Unit, Tampa General Hospital, Tampa, $\mathrm{FL}_{;}{ }^{4}$ Department of Obstetrics and Gynecology, University of Missouri at Kansas, Kansas \\ City, MO, USA
}

\section{Objective}

The primary outcome was to compare the diagnostic accuracy of neonatal small for gestational age (SGA) by the Hadlock and Fetal Medicine Foundation (FMF) charts in our cohort, followed by the ability to predict composite severe neonatal outcomes (SNO) in pregnancies with preterm prelabor rupture of membranes (PPROM).

\section{Methods}

This study was a secondary analysis of a prospective cohort of pregnancies with PPROM from 2015 to 2018, from 23 to 36 completed weeks of gestation. Sensitivity, specificity, and positive and negative predictive values for the primary and secondary outcomes of the Hadlock and FMF fetal charts were calculated. The discriminatory ability of each chart was compared using the area under the receiver's operating curves of clinical characteristics.

Results

Of the 106 women who met the inclusion criteria, 48 (45\%) were screened positive using the FMF fetal growth chart and $22(21 \%)$ were screened positive using the Hadlock chart. SGA was diagnosed in 12 infants (11\%). Both fetal growth charts had comparable diagnostic accuracies and were statistically significant predictors of SGA (Hadlock: area under the receiver operating characteristic curves [AUC], 0.76, risk ratio [RR], 7.6, 95\% confidence interval [CI], 2.5-23; and FMF: AUC, $0.76 \mathrm{RR}, 13.395 \% \mathrm{Cl}$ 1.8-99.3). Both growth standards were poor predictors of SNO.

\section{Conclusion}

The Hadlock and FMF fetal growth charts have a similar accuracy to predict SGA in pregnancies complicated by PPROM. The FMF fetal growth chart may result in a 2-fold increase in positive screens, potentially increasing fetal surveillance.

Keywords: Fetal growth restriction; Preterm prelabor rupture of membranes

\section{Introduction}

Small for gestational age (SGA) neonates refer to those with a birth weight $(B W)<10$ th percentile for gestational age [1]. Preterm prelabor rupture of membranes (PPROM) is defined as rupture of membranes before 37 weeks [2]. Both SGA and PPROM infants are at an increased risk for adverse outcomes [2-4], and SGA appears to complicate pregnancies with preterm labor and PPROM more often than uncomplicated pregnancies $[5,6]$. Therefore, the accurate prediction of SGA in pregnancies with PPROM will assist in creating surveillance protocols to reduce such adverse outcomes [7].
Received: 2020.09.06. Revised: 2020.11.09. Accepted: 2020.11.25. Corresponding author: Jose Duncan, MD

Department of Obstetrics and Gynecology, University of South Florida, 2 Tampa General Circ \# 6000 Tampa, Fl 33606, USA

E-mail: joserduncan@yahoo.com

https://orcid.org/0000-0001-8464-9744

Articles published in Obstet Gynecol Sci are open-access, distributed under the terms of the Creative Commons Attribution Non-Commercial License (http://creativecommons. org/licenses/by-nc/3.0/) which permits unrestricted non-commercial use, distribution, and reproduction in any medium, provided the original work is properly cited.

Copyright $\odot 2021$ Korean Society of Obstetrics and Gynecology 


\section{Obstetrics \& Gynecology Science}

Jose Duncan, et al. SGA detection in PPROM

The prevalence of fetal growth restriction (FGR) varies according to the definition used. In the United States, the most commonly used definition is an estimated fetal weight (EFW) $<10$ th percentile for gestational age; however, this definition will most likely include those that are constitutionally small [1]. Therefore, a new definition of FGR based on the Delphi criteria has been proposed by a group of international experts [8]. However, a recent study found that although the implementation of this definition was associated with a slight increase in the detection of adverse outcomes when compared to the definition endorsed by the American Congress of Obstetricians and Gynecologists (ACOG), both were poor predictors of adverse neonatal outcomes [9].

The ability to detect SGA by the EFW varies according to the formula used to calculate the EFW and the fetal growth chart utilized to assign the EFW percentile according to gestational age [10-12]. The Hadlock et al. [13] formula is the most commonly used method to calculate the EFW, as it appears to be an accurate predictor of the BW [10]. Multiple studies have attempted to identify the most accurate fetal growth chart, and most of their results suggest that none of the recently developed charts appears to be superior to the Hadlock et al. [14] growth chart in predicting SGA $[11,15,16]$.

Despite the accuracy of the EFW to predict BW, there is a discrepancy between fetal growth charts and neonatal growth charts, specifically in the preterm period $[17,18]$. This lack of correlation is most likely due to the inclusion of multiple risk factors that are associated with both FGR and prematurity [19]. To address this issue, the Fetal Medicine Foundation (FMF) developed a unified fetal and neonatal growth chart [20]. However, this chart has not been validated in specific obstetrical populations, such as PPROM.

We hypothesize that the FMF chart improves the detection of SGA and adverse outcomes in pregnancies with PPROM and FGR. Thus, our aim was to compare the detection accuracy for SGA using the Hadlock fetal versus the FMF fetal and neonatal weight chart in pregnancies complicated by PPROM.

\section{Materials and methods}

This study was a secondary analysis of a prospective cohort of pregnancies complicated by PPROM managed in an academic hospital affiliated to the University of Tennessee Health
Science Center from October 2015 to June 2018.

Inclusion criteria included singleton pregnancies of mothers aged 13-46 years, from 23 weeks to 36 completed weeks of gestation, with an EFW within 2 weeks prior to delivery. We excluded pregnancies with complex fetal anomalies, those with fetal demise, and those with incomplete data. In our institution, the diagnosis of PPROM is confirmed by the visualization of amniotic fluid passing from the cervical canal and pooling in the vagina. When diagnosis is not confirmed, a $\mathrm{pH}$ test of the vaginal fluid showing an arborization (ferning) of the dried vaginal fluid test, and the amniotic fluid was measured. If the diagnosis is uncertain, the amniodye test was done to confirm or exclude the final diagnosis.

All included participants signed an informed consent form. Demographic and clinical characteristics including the maternal age, parity, self-reported race, body mass index at presentation, gestational age at PPROM, presence of hypertension, diabetes, chorioamnionitis, latency, gestational age at delivery, and BW were extracted from maternal medical records. Neonatal outcomes such as the presence of respiratory distress (RDS), grade III-IV intraventricular hemorrhage

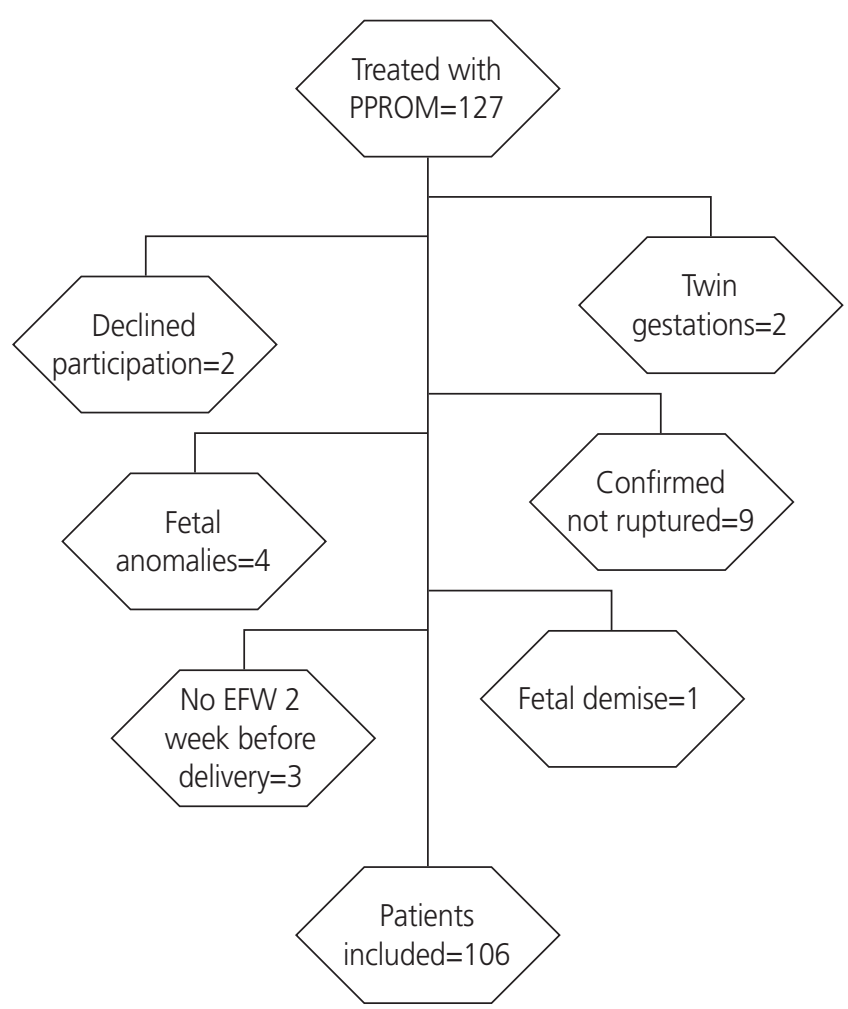

Fig. 1. Study population. PPROM, preterm prelabor rupture of membranes; EFW, estimated fetal weight. 


\title{
Obstetrics \& Gynecology Science
}

\author{
Vol. 64, No. 3, 2021
}

(IVH), neonatal sepsis, necrotizing enterocolitis (NEC) stages 2 and 3 , and neonatal death, were obtained from the infants' electronic medical records.

The primary outcome of this study was to calculate and compare the diagnostic accuracy for neonatal SGA using the Hadlock and FMF charts in our cohort. SGA was suspected when FGR was detected (EFW <10th percentile) using the Hadlock or FMF chart. Neonatal SGA was diagnosed with a BW <10th percentile based on the Alexander et al. [21] BW chart as this is used in the study's institution.

The secondary outcome was to compare the ability of FGR on both growth charts to predict a composite of severe neonatal outcomes (SNO) when at least one of the following were diagnosed: RDS, IVH, NEC, neonatal sepsis, and perinatal death. We also calculated the rate accuracy of the FMF fetal growth chart to diagnose SGA using the proposed FMF neonatal BW chart [22]. The rate of SGA was also obtained using this approach. In this study, RDS was defined as having a clinical and radiologic evidence of surfactant deficiency [23], and grade III and IV IVH were defined according to the Papile et al. [24] classification. NEC stages 2 and 3 were defined according to Bell's staging [25], neonatal sepsis was confirmed with positive blood cultures, and perinatal death comprised deaths occurring from 20 weeks of gestation to the first year of life. Gestational age was assessed using the ACOG guidelines [26]. We defined low amniotic fluid as a deep vertical pocket (DVP) $<2 \mathrm{~cm}$.

Ultrasounds (USs) were performed using a GE Voluson E8 (GE Healthcare, Chicago, IL, USA) with a maternal-fetal medicine fellow or an obstetric and gynecology resident. Both residents and fellows received training by certified sonographers and maternal-fetal medicine specialists. The EFW was calculated every 2 weeks using the Hadlock et al.'s formula [13].

\section{Statistical analysis}

Statistical analysis was performed using STATA 15 (StataCorp., College Station, TX, USA). Categorical data were compared using $\chi^{2}$ test or Fischer's exact text where appropriate and were presented as numbers (\%). For continuous variables,

Table 1. Obstetrical characteristics of SGA

\begin{tabular}{|c|c|c|c|}
\hline Characteristic & Non-SGA $(n=94)$ & SGA $(n=12)$ & $P$-value \\
\hline Age (yr) & $25.0(21.0-32.0)$ & $24.5(19.5-29.0)$ & 0.55 \\
\hline Primiparous & $33(35.1)$ & $4(33.3)$ & 0.90 \\
\hline Ethnicity & & & 0.97 \\
\hline African American & $79(74.5)$ & $11(10.4)$ & \\
\hline Caucasian & $8(7.5)$ & $1(0.9)$ & \\
\hline Asian & $6(5.7)$ & 0 & \\
\hline Hispanic & $1(0.9)$ & 0 & \\
\hline Body mass index $\left(\mathrm{kg} / \mathrm{m}^{2}\right)$ & $32.0(25.7-38.3)$ & $29.3(23.8-30.7)$ & 0.07 \\
\hline Chronic hypertension & 10 & 0 & - \\
\hline Diabetes mellitus & 6 & 0 & - \\
\hline Gestational diabetes & 7 & 0 & - \\
\hline Gestational age at PPROM (wk) & $29.6(26.4-32.6)$ & $32.3(25.0-32.9)$ & 0.75 \\
\hline Gestational age at ultrasound (wk) & $30.8(27.6-33.2)$ & $33.2(32.0-33.6)$ & 0.17 \\
\hline Ultrasound $<7$ days from delivery & $84(89)$ & $12(100)$ & 0.57 \\
\hline Latency (day) & $4.5(1-10)$ & $4.5(1-10)$ & 0.77 \\
\hline Gestational age at delivery (wk) & $31.1(28.1-33.3)$ & $33.6(32.7-34.0)$ & 0.11 \\
\hline Cesarean delivery & $33(35.1)$ & $5(41.7)$ & 0.66 \\
\hline Birth weight (g) & $1,560(1,140-2,140)$ & $1,570(1,390-1,860)$ & 0.55 \\
\hline Deep vertical pocket $<2(\mathrm{~cm})$ & $35(14)$ & $3(8)$ & 0.55 \\
\hline
\end{tabular}

Data presented as number (\%) or median (interquartile range).

SGA, small for gestational age; PPROM, preterm prelabor rupture of membranes. 


\section{Obstetrics \& Gynecology Science}

Jose Duncan, et al. SGA detection in PPROM

the distribution of the data was judged by the skewness and kurtosis tests. The Wilcoxon rank sum test was used for statistical analysis when the data were not normally distributed; continuous variables were presented as median and interquartile ranges.

The risk ratios (RRs) and 95\% confidence interval $(\mathrm{Cl})$ were estimated using the non-SGA group as the reference group. Sensitivity, specificity, and positive and negative predictive values for the primary and secondary outcomes using these

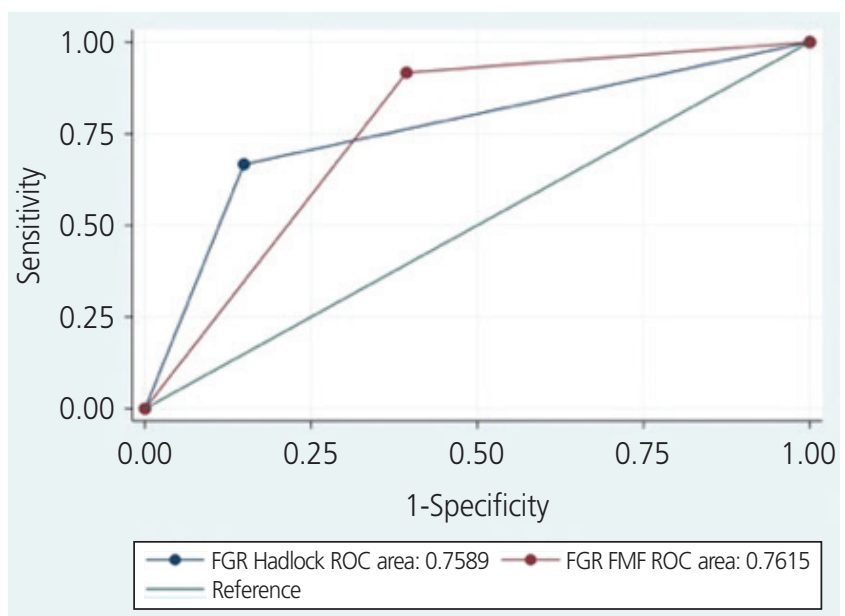

Fig. 2. Area under the receiver operating characteristics (ROC) curves for the prediction of small for gestational age by fetal growth restriction Fetal Medicine Foundation (FGR FMF) and the Hadlock fetal growth chart (FGR Hadlock). fetal weight standards were calculated. The discriminatory ability of each fetal growth chart was compared using the area under the receiver operating characteristic curves (AUC). A $P$-value $<0.05$ was consider significant.

\section{Results}

A total of 127 women were treated for PPROM in our institution during the study period. Of these, 106 women met the inclusion criteria and were included in the analysis (Fig. 1). All of our patients completed at least one course of betamethasone prior to delivery. Among them, 48 (45\%) were screened positive for SGA using the FMF fetal growth chart and 22 (21\%) were screened positive using the Hadlock method (RR, 7.6, 95\% Cl, 2.4-24.3, $P<0.001$ ). SGA was diagnosed in 12 infants (11\%). Of the 106 women included, 96 had an US within 7 days of delivery; 3 of the 12 infants in the SGA group had low amniotic fluid, and 35 of 90 participants (4 did not have documented DVP) had a low amniotic fluid measurement in the non-SGA group. The EFW percentage within $10 \%$ of the BW was comparable in those with SGA and those without SGA (75\% vs. $65 \% ; P=0.73$ ). Demographics and obstetrical characteristics did not significantly differ among infants with SGA and those without SGA (Table 1). Both fetal growth charts had similar prediction accuracy and were statistically significant predictors of SGA (Hadlock:

Table 2. Detection of SGA and a composite of SNO and fetal growth restriction by the Hadlock and FMF weight charts in pregnancies complicated by PPROM $(n=106)$

\begin{tabular}{|c|c|c|c|c|c|c|}
\hline \multirow[b]{2}{*}{ Characteristic } & \multicolumn{3}{|c|}{ Detection of SGA $(n=12)$} & \multicolumn{3}{|c|}{ Detection of SNO $(n=42)$} \\
\hline & Hadlock $^{\text {a) }}$ & FMF $^{\text {b) }}$ & $\begin{array}{c}\text { SGA by FMF fetal } \\
\text { and neonatal charts }\end{array}$ & Hadlock $^{\text {a) }}$ & $\mathrm{FMF}^{\mathrm{b})}$ & FMF neonatal chart ${ }^{\mathrm{d})}$ \\
\hline Sensitivity & 66.7 & 91.7 & 74.4 & 19.0 & 52.3 & 47.6 \\
\hline Specificity & 85.1 & 60.6 & 74.6 & 78.1 & 59.4 & 64.0 \\
\hline Positive predictive value & 36.4 & 22.9 & 66.7 & 54.7 & 56.6 & 57.6 \\
\hline Negative predictive value & 95.2 & 98.3 & 81.0 & 39.6 & 39.6 & 39.6 \\
\hline Positive screening tests & 21.0 & 45.0 & 45.0 & - & - & - \\
\hline Prevalence of SGA & 11.2 & 11.2 & 40.6 & - & - & - \\
\hline
\end{tabular}

Data presented as percentage.

SGA, small for gestational age; SNO, severe neonatal outcomes; FMF, Fetal Medicine Foundation; PPROM, preterm prelabor rupture of membranes.

a) Performance of the Hadlock chart to detect SGA based on the Alexander birthweight chart and to detect the composite of SNO; ${ }^{\text {b) Perfor- }}$ mance of the FMF fetal chart to detect SGA based on the Alexander birthweight chart and to detect the composite of SNO; 'Detection of SGA by the FMF fetal and neonatal weight charts; ${ }^{\text {d) }}$ Detection of SNO by the neonatal FMF weight chart. 


\title{
Obstetrics \& Gynecology Science
}

\author{
Vol. 64, No. 3, 2021
}

AUC, 0.76, RR, 7.6, 95\% Cl, 2.5-23, P<0.001; FMF: AUC, $0.76 \mathrm{RR}, 13.395 \% \mathrm{Cl} 1.8-99.3, P \leq 0.001)$. The diagnostic accuracy for SGA using the Hadlock fetal growth chart was similar to that of the FMF fetal growth chart (Fig. 2). The sensitivity, specificity, and positive and negative predictive values are presented in Table 2. Four SGA neonates were lost using the Hadlock fetal growth chart, and only one SGA with the FMF fetal growth chart.

For our secondary outcomes, FGR by the Hadlock and FMF fetal growth charts had poor prediction abilities for the composite of SNO (Hadlock: AUC, 0.51, RR, $0.84,95 \%$ Cl, 0.5-1.6 vs. FMF: ROC, $0.56, \mathrm{RR}, 1.6,95 \% \mathrm{Cl}, 0.7-3.5, P=0.23$ ) (Fig. 3). SNO occurred in only 2 of the 12 SGA infants (17\%) vs. 40 $(42 \%)$ of the infants with appropriate weight for gestational age (RR, 0.4, 95\% Cl, 0.1-1.4, P=0.012). Both SGA infants with SNO had RDS, and one was diagnosed with NEC.

SGA would have been diagnosed in $40 \%$ of our participants if both the fetal and neonatal FMF growth charts were utilized vs. 11\% using the Alexander BW growth chart (RR, 3.3, 95\% Cl, 2.3-4, $P \leq 0.001)$. The FMF fetal chart was a statistically significant predictor of SGA defined by the FMF neonatal standard (AUC, 0.74, RR, 3.5, 95\% Cl, 2.0-6.2, $P \leq 0.001)$, but the FMF neonatal standard was not an accurate predictor for the composite of neonatal outcomes (AUC, 0.56, RR, 1.3, 95\% Cl, 0.8-2.1, P=0.23). The sensitivity, specificity, and positive and negative predictive values for FMF standards are presented in Table 2.

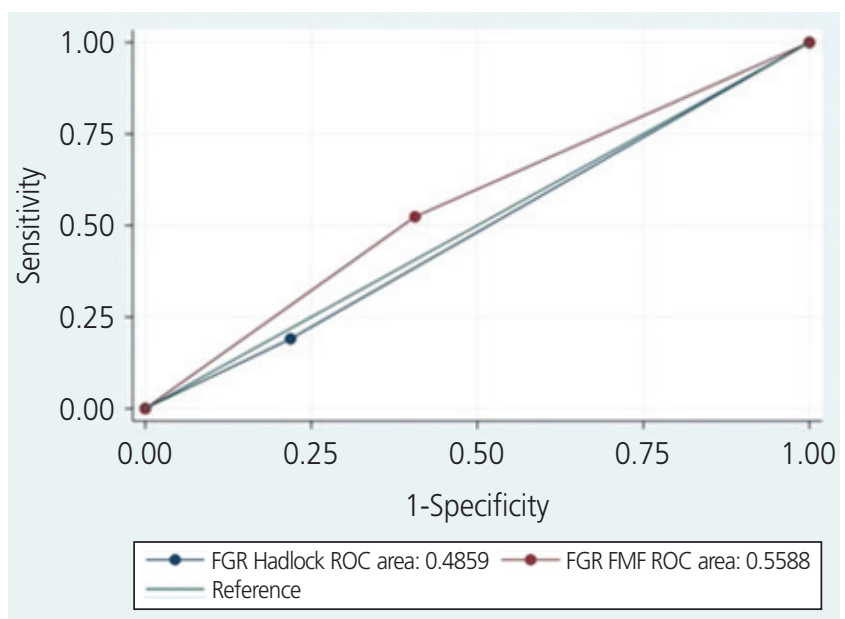

Fig. 3. Area under the receiver operating characteristics (ROC) curves for the prediction of severe neonatal outcomes by fetal growth restriction Fetal Medicine Foundation (FGR FMF) and the Hadlock fetal growth chart (FGR Hadlock).

\section{Discussion}

In this cohort study of pregnancies with PPROM, SGA was diagnosed in $11 \%$ of the infants, and we found that the Hadlock and FMF fetal growth charts have similar prediction accuracy for SGA (both with an AUC of 0.76 ). The FMF has a higher sensitivity (91.7\% vs. 66.7\%) and the Hadlock chart has a better specificity for the detection of SGA ( $85.1 \%$ vs. $60.6 \%)$. However, both growth charts had a low positive predictive value ( $21 \%$ for Hadlock and $45 \%$ for FMF). Moreover, the rate of FGR with the FMF was $45 \%$ vs. $21 \%$ with the Hadlock chart. Both fetal growth standards were not statistically significant predictors of SNO, but only 2 of the 12 SGA neonates experienced SNO.

We had previously reported the rate of FGR $(21 \%)$ in this cohort of pregnancies with PPROM [27]. The rate of FGR and SGA in PPROM depends on the definition, population, gestational age, and weight chart utilized. In a large multicenter randomized trial that included more than 1,800 pregnancies with PPROM from 34-36 weeks of gestation randomized to immediate delivery versus expectant management, an SGA rate of $3.6 \%$ was reported [28]. Another large multicenter observational study from France that included 702 gestations, evaluating the effect of the duration of latency in pregnancies with PPROM from 24-32 weeks, only reported on those with BWs $<3$ rd percentile (7.5\% of their cohort) [29]. Neither of these studies described which weight charts were used. A small cohort of 69 pregnancies from Italy with PPROM between 24-31 weeks reported rates of FGR of 39\% and SGA rates of $26 \%$ [30]; they used an Italian neonatal BW chart [31].

However, the question of what chart to use to predict SGA still remains unclear. In theory, the optimal fetal growth chart will not only accurately detect SGA but will also identify those with adverse outcomes among those to screen positive. A retrospective cohort from New Mexico that included over 1,500 women concluded that the Hadlock fetal growth chart was superior to the Eunice Kennedy Shriver National Institute of Child Health and Human Development (NICHD) race/ethnicity-specific standard in predicting both neonatal morbidity and SGA [15]. Another single-center retrospective study from Detroit, which included 3,437 African American mothers, reported that the NICHD race-specific weight standard was superior to the Hadlock and other weight charts in predicting adverse outcomes in SGA infants [11]. Another 


\section{Obstetrics \& Gynecology Science}

Jose Duncan, et al. SGA detection in PPROM

study from 2 institutions compared the Hadlock fetal chart with the NTERGROWTH-21st Century growth and concluded that although the Hadlock chart may have a superior ability to predict SGA, none of these charts were accurate predictors of adverse neonatal outcomes [16].

In our study, the Hadlock and FMF growth charts were not accurate predictors of SNO. However, we recognize the lack of power to detect such differences using a sample of only 12 SGA infants.

Another limitation in our study is that we only assessed 2 out of the many fetal growth standards described in the literature [32-34]. Nevertheless, we included the Hadlock chart, as it is used in our institution, and similar studies have compared 2 modalities.

We also acknowledge that the number of our study participant is inferior to that in other studies $[11,15,16]$; it was carried out in a single institution, with a study population composed mostly of African Americans (85\%). Therefore, our results may not be generalizable to other populations. However, we evaluated the accuracy of these growth charts only in pregnancies complicated by PPROM, which only complicates 2-3\% of the obstetrical population [35]. Lastly, the accuracy of our US measurement could be questioned for a couple of reasons. First, low amniotic fluid is common in pregnancies with PPROM, and this has been shown to decrease the accuracy of the EFW, and second, the US ex- aminations were performed by trainees. Nevertheless, we previously validated the accuracy of the EFW measured in this study population [36], and the presence of low amniotic fluid was comparable in those with SGA and those without (Table 3).

Our strengths include the following: for our first outcome, we included the detection of SGA in a population where this diagnosis has been associated with an increased risk of infant morbidity and mortality $[37,38]$. In addition, the evaluated adverse neonatal outcomes in our secondary outcomes have been previously validated and are known to cause severe morbidity and mortality in premature infants $[37,39]$. Lastly, both charts studied were derived from the Hadlock formula, and the same has been validated in our PPROM population [36], and the EFW in this population has been shown to be a significant predictor of adverse perinatal outcomes [40].

In conclusion, it appears that both the Hadlock and FMF fetal growth charts have similar prediction accuracy for SGA in pregnancies complicated by PPROM, but the use of the FMF fetal growth chart may result in a 2-fold increase in positive screens, potentially increasing fetal surveillance in these patients. We acknowledge the need for more diverse studies to corroborate our results and identify the optimal fetal growth standard to detect SGA and severe adverse neonatal outcomes in pregnancies with PPROM.

Table 3. Small gestational infants in our study

\begin{tabular}{lccccccc}
\hline $\begin{array}{l}\text { Small } \\
\text { gestational } \\
\text { infants }\end{array}$ & $\begin{array}{c}\text { Body mass } \\
\text { index } \\
\left(\mathbf{k g} / \mathbf{m}^{2}\right)\end{array}$ & $\begin{array}{c}\text { GA at } \\
\text { ultrasound }\end{array}$ & EFW & $\begin{array}{c}\text { Amniotic fluid } \\
\text { deep vertical } \\
\text { pocket }\end{array}$ & BW (g) & $\begin{array}{c}\text { GA at } \\
\text { delivery }\end{array}$ & $\begin{array}{c}\text { Mean (\%) } \\
\text { difference of } \\
\text { BW-EFW }\end{array}$ \\
\hline 1 & 41.8 & 33.0 & 1,620 & 2.14 & 1,330 & 33.4 & 21.80 \\
2 & 17.8 & 24.0 & 596 & 0 & 480 & 24.1 & 24.17 \\
3 & 24.8 & 34.0 & 2,004 & 3.06 & 1,890 & 34.1 & 6.03 \\
4 & 29.8 & 33.5 & 1,676 & 2.49 & 1,640 & 34.0 & 2.20 \\
5 & 23.8 & 32.6 & 1,879 & 3.74 & 1,850 & 33.6 & 1.57 \\
6 & 32.1 & 34.0 & 2,413 & 3.48 & 1,920 & 34.0 & 25.68 \\
7 & 30.0 & 32.5 & 1,351 & 2.00 & 1,450 & 33.0 & 6.83 \\
8 & 23.8 & 24.1 & 547 & 0 & 530 & 25.0 & 3.21 \\
9 & 29.3 & 31.6 & 1,458 & 2.50 & 1,500 & 32.4 & 2.80 \\
10 & 29.3 & 33.5 & 1,700 & 2.15 & 1,740 & 34.0 & 2.30 \\
11 & 31.3 & 33.5 & 1,773 & 3.50 & 1,870 & 34.1 & 5.19 \\
12 & 22.5 & 33.6 & 1,486 & 0 & 1,460 & 33.6 & 1.78 \\
\hline
\end{tabular}

GA, gestational age; EFW, estimated fetal weight; BW, birth weight. 


\section{Obstetrics \& Gynecology Science}

Vol. 64, No. 3, 2021

\section{Conflict of interest}

No potential conflict of interest relevant to this article was reported.

\section{Ethical approval}

The University of Tennessee Health Science Center Institutional Review Board approved our study protocol (XP1504083).

\section{Patient consent}

All included participants signed informed consent.

\section{Funding information}

None.

\section{References}

1. American College of Obstetricians and Gynecologists' Committee on Practice Bulletins-Obstetrics and the Society for Maternal-Fetal Medicine. ACOG practice bulletin No. 204: fetal growth restriction. Obstet Gynecol 2019;133:e97-109.

2. American College of Obstetricians and Gynecologists' Committee on Practice Bulletins-Obstetrics. Prelabor rupture of membranes: ACOG practice bulletin, number 217. Obstet Gynecol 2020;135:e80-97.

3. Kim WJ, Han YS, Ko HS, Park IY, Shin JC, Wie JH. Antenatal corticosteroids and outcomes of preterm smallfor-gestational-age neonates in a single medical center. Obstet Gynecol Sci 2018;61:7-13.

4. Kim SY, Kwon HS, Lee J, Sul AR, Ko HS, Yang Jl. Questionnaire survey on the management of pregnant women with preterm premature rupture of membranes. Obstet Gynecol Sci 2020;63:286-92.

5. Hediger ML, Scholl TO, Schall Jl, Miller LW, Fischer RL. Fetal growth and the etiology of preterm delivery. Obstet Gynecol 1995;85:175-82.
6. Monier I, Ancel PY, Ego A, Guellec I, Jarreau PH, Kaminski $\mathrm{M}$, et al. Gestational age at diagnosis of early-onset fetal growth restriction and impact on management and survival: a population-based cohort study. BJOG 2017;124:1899-906.

7. Selvaratnam RJ, Davey MA, Anil S, McDonald SJ, Farrell T, Wallace EM. Does public reporting of the detection of fetal growth restriction improve clinical outcomes: a retrospective cohort study. BJOG 2020;127:581-9.

8. Gordijn SJ, Beune IM, Thilaganathan B, Papageorghiou A, Baschat AA, Baker PN, et al. Consensus definition of fetal growth restriction: a Delphi procedure. Ultrasound Obstet Gynecol 2016;48:333-9.

9. Molina LCG, Odibo L, Zientara S, Običan SG, Rodriguez A, Stout $M$, et al. Validation of the Delphi procedure consensus criteria for defining fetal growth restriction. Ultrasound Obstet Gynecol 2020;56:61-6.

10. Hammami A, Mazer Zumaeta A, Syngelaki A, Akolekar $\mathrm{R}$, Nicolaides $\mathrm{KH}$. Ultrasonographic estimation of fetal weight: development of new model and assessment of performance of previous models. Ultrasound Obstet Gynecol 2018;52:35-43.

11. Kabiri D, Romero R, Gudicha DW, Hernandez-Andrade E, Pacora P, Benshalom-Tirosh N, et al. Prediction of adverse perinatal outcomes by fetal biometry: a comparison of customized and population-based standards. Ultrasound Obstet Gynecol 2020;55:177-88.

12. Blue NR, Savabi M, Beddow ME, Katukuri VR, Fritts CM, Izquierdo LA, et al. The Hadlock method is superior to newer methods for the prediction of the birth weight percentile. J Ultrasound Med 2019;38:587-96.

13. Hadlock FP, Harrist RB, Sharman RS, Deter RL, Park SK. Estimation of fetal weight with the use of head, body, and femur measurements--a prospective study. Am J Obstet Gynecol 1985;151:333-7.

14. Hadlock FP, Harrist RB, Martinez-Poyer J. In utero analysis of fetal growth: a sonographic weight standard. Radiology 1991;181:129-33.

15. Blue NR, Beddow ME, Savabi M, Katukuri VR, Chao CR. Comparing the Hadlock fetal growth standard to the Eunice Kennedy Shriver National Institute of Child Health and Human Development racial/ethnic standard for the prediction of neonatal morbidity and small for gestational age. Am J Obstet Gynecol 2018;219:474.e112. 


\section{Obstetrics \& Gynecology Science}

Jose Duncan, et al. SGA detection in PPROM

16. Nwabuobi C, Odibo L, Camisasca-Lopina H, Leavitt K, Tuuli M, Odibo AO. Comparing INTERGROWTH-21st Century and Hadlock growth standards to predict small for gestational age and short-term neonatal outcomes. J Matern Fetal Neonatal Med 2020;33:1906-12.

17. Tamura RK, Sabbagha RE, Dooley SL, Vaisrub N, Socol ML, Depp R. Real-time ultrasound estimations of weight in fetuses of diabetic gravid women. Am J Obstet Gynecol 1985;153:57-60.

18. GRIT Study Group. A randomised trial of timed delivery for the compromised preterm fetus: short term outcomes and Bayesian interpretation. BJOG 2003;110:27-32.

19. Gardosi JO. Prematurity and fetal growth restriction. Early Hum Dev 2005;81:43-9.

20. Nicolaides KH, Wright $D$, Syngelaki A, Wright A, Akolekar R. Fetal Medicine Foundation fetal and neonatal population weight charts. Ultrasound Obstet Gynecol 2018;52:44-51.

21. Alexander GR, Himes JH, Kaufman RB, Mor J, Kogan M. A United States national reference for fetal growth. Obstet Gynecol 1996;87:163-8.

22. Bischoff A, Calvo-Garcia MA, Baregamian N, Levitt MA, Lim FY, Hall J, et al. Prenatal counseling for cloaca and cloacal exstrophy-challenges faced by pediatric surgeons. Pediatr Surg Int 2012;28:781-8.

23. Duncan JR, Tobiasz AM, Dorsett KM, Aziz MM, Thompson RE, Bursac $Z$, et al. Fetal pulmonary artery acceleration/ejection time prognostic accuracy for respiratory complications in preterm prelabor rupture of membranes. J Matern Fetal Neonatal Med 2020;33:2054-8.

24. Papile LA, Burstein J, Burstein R, Koffler H. Incidence and evolution of subependymal and intraventricular hemorrhage: a study of infants with birth weights less than 1,500 gm. J Pediatr 1978;92:529-34.

25. Bell MJ, Ternberg JL, Feigin RD, Keating JP, Marshall R, Barton $L$, et al. Neonatal necrotizing enterocolitis. Therapeutic decisions based upon clinical staging. Ann Surg 1978;187:1-7.

26. Reddy UM, Abuhamad AZ, Levine D, Saade GR, Fetal Imaging Workshop Invited Participants. Fetal imaging: executive summary of a joint Eunice Kennedy Shriver National Institute of Child Health and Human Development, Society for Maternal-Fetal Medicine, American Institute of Ultrasound in Medicine, American College of Obstetricians and Gynecologists, American College of Radiology, Society for Pediatric Radiology, and Society of Radiologists in Ultrasound Fetal Imaging workshop. Obstet Gynecol 2014;123:1070-82.

27. Duncan JR, Dorsett KM, Vilchez G, Schenone MH, Mari $G$. Uterine artery pulsatility index for the prediction of obstetrical complications in preterm prelabor rupture of membranes. J Matern Fetal Neonatal Med 2019 Dec 16 [Epub]. http://doi.org/10.1080/14767058.2019.1702961.

28. Morris JM, Roberts CL, Bowen JR, Patterson JA, Bond $D M$, Algert CS, et al. Immediate delivery compared with expectant management after preterm pre-labour rupture of the membranes close to term (PPROMT trial): a randomised controlled trial. Lancet 2016;387:444-52.

29. Lorthe E, Ancel PY, Torchin H, Kaminski M, Langer B, Subtil $D$, et al. Impact of latency duration on the prognosis of preterm infants after preterm premature rupture of membranes at 24 to 32 weeks' gestation: a national population-based cohort study. J Pediatr 2017;182:4752.e2.

30. Spinillo A, Montanari L, Sanpaolo P, Bergante C, Chiara A, Fazzi E. Fetal growth and infant neurodevelopmental outcome after preterm premature rupture of membranes. Obstet Gynecol 2004;103:1286-93.

31. Parazzini F, Cortinovis I, Bortolus R, Zanardo V. Birth weight of infants born between the 23rd and $42 \mathrm{nd}$ gestational week in Italy. Pediatr Med Chir 1998;20:93-7.

32. Papageorghiou AT, Ohuma EO, Altman DG, Todros T, Cheikh Ismail L, Lambert A, et al. International standards for fetal growth based on serial ultrasound measurements: the Fetal Growth Longitudinal Study of the INTERGROWTH-21st Project. Lancet 2014;384:869-79.

33. Buck Louis GM, Grewal J, Albert PS, Sciscione A, Wing DA, Grobman WA, et al. Racial/ethnic standards for fetal growth: the NICHD Fetal Growth Studies. Am J Obstet Gynecol 2015;213:449.e1-41.

34. Gardosi J, Francis A, Turner S, Williams M. Customized growth charts: rationale, validation and clinical benefits. Am J Obstet Gynecol 2018;218:S609-18.

35. Mercer BM. Preterm premature rupture of the membranes. Obstet Gynecol 2003;101:178-93.

36. Duncan JR, Schenone C, Dorset KM, Goedecke PJ, Tobiasz AM, Meyer NL, et al. Estimated fetal weight accuracy in pregnancies with preterm prelabor rupture of membranes by the Hadlock method. J Matern Fetal Neonatal Med 2020 May 22 [Epub]. http://doi.org/10.10 


\section{Obstetrics \& Gynecology Science}

Vol. 64, No. 3, 2021

80/14767058.2020.1769593.

37. EXPRESS Group; Fellman V, Hellström-Westas L, Norman $M$, Westgren $M$, Källén $K$, et al. One-year survival of extremely preterm infants after active perinatal care in Sweden. JAMA 2009;301:2225-33.

38. Grace MR, Dotters-Katz S, Varner MW, Boggess K, Manuck TA. Birthweight extremes and neonatal and childhood outcomes after preterm premature rupture of membranes. Am J Perinatol 2016;33:1138-44.
39. Ancel PY, Goffinet F; EPIPAGE-2 Writing Group, Kuhn P, Langer $B$, Matis J, et al. Survival and morbidity of preterm children born at 22 through 34 weeks' gestation in France in 2011: results of the EPIPAGE-2 cohort study. JAMA Pediatr 2015;169:230-8.

40. Duncan JR, Dorsett KM, Aziz MM, Bursac Z, Cleves MA, Talati AJ, et al. Estimated fetal weight and severe neonatal outcomes in preterm prelabor rupture of membranes. J Perinat Med 2020;48:687-93. 